\title{
Predictors of quality of life in patients with end-stage renal disease on hemodialysis
}

This article was published in the following Dove Press journal: International Journal of Nephrology and Renovascular Disease 3 September 2015

Number of times this article has been viewed

\section{Marc M Saad' \\ Youssef El Douaihy' \\ Christine Boumitri' \\ Chetana Rondla ${ }^{2}$ \\ Elias Moussaly' \\ Magda Daoud' \\ Suzanne E El Sayegh ${ }^{3}$}

Internal Medicine, Staten Island University Hospital, Staten Island, NY, ${ }^{2}$ Nephrology, Emory University Hospital, Atlanta, GA, ${ }^{3}$ Nephrology, Staten Island University Hospital, Staten Island, NY, USA
Correspondence: Marc M Saad Internal Medicine, Staten Island University Hospital, 475 Seaview Avenue, Staten Island, NY 10305, USA

Emailmsaad2@nshs.edu
Background: Assessment of quality of life (QOL) of end-stage renal disease (ESRD) patients (physical, mental, and social well-being) has become an essential tool to develop better plans of care. Objective of this study is to determine which demographic and biochemical parameters correlate with the QOL scores in patients with ESRD on hemodialysis (HD) using Kidney Disease QOL-36 surveys (KDQOL).

Methods: A retrospective chart review of all ESRD patients who underwent HD at an outpatient center. The five components of the KDQOL were the primary end points of this study (burden of kidney disease, symptoms and problems, effects of kidney disease on daily life, mental component survey, and physical component survey). Scores were grouped into three categories (below average, average, and above average). In addition to demographics (age, sex, and race), the independent variables such as weight gain, number of years on dialysis, urea reduction ratio, calcium, phosphorus, parathyroid hormone, albumin, and hemoglobin in the serum were collected. Chi-square analysis for dependent variables and the nominal independent variables was used, and analysis of variance analysis was used for continuous independent variables. Ordinal regression using PLUM (polytomous universal model) method was used to weigh out possible effects of confounders.

Results: The cohort size was 111 patients. Mean age was $61.8( \pm 15.5)$ years; there were more males than females $(64.9 \%$ vs $35.1 \%)$, the mean time-on-dialysis at the time of the study was 4.3 (4.8) years. Approximately two-thirds of the responses on all five domains of the questionnaire ranked average when compared to the national numbers. The remainders were split between above average (20.6\%) and below average (13.4\%). In our cohort, no relationships were statistically significant between the five dependent variables of interest and the independent variables by chi-square- and $t$-test analyses. This was further confirmed by regression analysis. Of note, sex carried the strongest statistical significance (with a $P$-value of 0.16 ) as a predictor of "the burden of kidney disease on daily life" in ordinal regression.

Conclusion: Prior studies have shown variables such as serum phosphate level, intradialytic weight gain, and dialysis adequacy are associated with lower KDQOL scores; however, this was not evident in our analysis likely due to smaller sample size. Larger size studies are required to better understand the predictors of QOL in ESRD patients on HD.

Keywords: quality of life, end-stage renal disease, hemodialysis, metabolic profile

\section{Background}

Health, as defined by World Health Organization, is a state of complete physical, mental, and social well-being, and not merely the absence of disease or infirmity. ${ }^{1}$ Thus, in chronic diseases, the quality of life (QOL), which describe the patient health, is an essential scale for assessing the success of a treatment. Health-related QOL (HRQOL) 
is the subjective perception of the illness and its treatment on the physical, psychological, and social well-being. ${ }^{1,2}$

Patients with end-stage renal disease (ESRD) on hemodialysis (HD) experience the heavy burden of dialysis treatment through its physical dependence, mental influence, and the myriad symptoms of ESRD.

Many studies showed that ESRD patients on HD have poor HRQOL when compared with the general population, ${ }^{1,3,4}$ and it is highly associated with malnutrition. ${ }^{3}$ In addition, it is influenced by the types of renal replacement modality as kidney-transplanted patient have better QOL compared to dialysis patients. Wu et al in a prospective study found that HD patients had greater improvement in Short-Form 36 (SF-36) domain scores than peritoneal dialysis patients. ${ }^{5}$

Lower HRQOL is associated with the higher rate of hospitalizations and worse survival among dialysis patients, ${ }^{1}$ serving as a prognostic measure and predictor for survival. ${ }^{4}$ In particular, physical and mental health score are independently associated with morbidity and mortality. 3,4

The assessment of QOL of ESRD patients (physical, mental, and social well-being) has become an essential tool to develop better plans of care (preventive and treatment strategies) and a recommended routine clinical practice., ${ }^{1,6}$

Comorbid disorders are prevalent in chronic kidney disease patients, such as depression (30\% of ESRD patients), sexual dysfunction (nearly half of ESRD), and problems with sleep (up to $40 \%-80 \%$ ), ranging from insomnia, sleep apnea to restless leg syndrome, and are associated with mortality as well as QOL, ${ }^{7}$ all of which contribute to worse QOL.

Nutritional status is an important determinant. Body mass index, cholesterol, serum albumin, hemoglobin, and dietary intakes correlate with QOL scores and strongly predict the longevity in this patient population. ${ }^{3,4}$ Nutritional deficits and diet restrictions are frequent problems that imply negative health outcomes with high mortality risk and QOL deterioration. $^{7}$

Poor physical health and mental health are highly reported in this population and the latter is an independent risk factor for mortality in patients on dialysis. ${ }^{1,4}$ Physical function decline is a prevalent feature in HD population and seems to affect negatively QOL even for younger HD patients. Moreover, physical function constitutes a positive impact on all domains of QOL emphasizing the major interest in physical rehab as a domain of interest. Thus, maintaining physical performance and independence is crucial to their overall being. Preserving residual kidney function, but not early dialysis treatment as found in the IDEAL (initiating dialysis early and late) study, have also been associated with better scores. ${ }^{7}$ Other factors are HD duration, age, and ethnicity. ${ }^{3}$ Most of these associations are reported based on observational studies, and HRQOL domains lack randomized control trials describing the potential causality, effect, or interventions in those areas. ${ }^{1}$

The objective of this study is to determine which demographic and biochemical parameters correlate with the QOL scores in patients with ESRD on HD in Staten Island Rehab Center using Kidney Disease QOL-36 surveys (KDQOL).

\section{Materials and methods}

\section{Patients and study design}

This is a retrospective cross-sectional analysis conducted at a single-outpatient HD center. After obtaining approval from the North Shore-LIJ review board, patients who received HD between March 2013 and July 2013 and who filled the KDQOL, validated and widely used questionnaire, were selected. The five components of the KDQOL were the primary end points of this study (burden of kidney on disease, symptoms and problems, effects of kidney disease on daily life, mental component survey [MCS], and physical component survey [PCS]). The end points were recorded in the score and category (below average, average, and above average) ways. In addition to demographics (age and ethnicity), the independent variables "weight gain between treatment", "dialysis vintage", and "levels of urea reduction ratio (URR), calcium, phosphorus, parathyroid hormone (PTH), albumin, and hemoglobin in the serum" were collected in an attempt to identify possible predictors of the categorized results of the different components of the questionnaire. Chi-square analysis was initially used to depict relationships between the dependent variables and the nominal independent variables; similarly, analysis of variance analysis was used for continuous independent variables. Ordinal regression using the PLUM method was used to plot all variables to weight out the possible effects of confounders. Statistical significance was defined at $P$-value $<0.05$.

\section{SF-36 questionnaire}

The QOL was assessed using Kidney Disease Quality of Life-Short-Form 36-items surveys (KDQOL-SF36). This is a self-administered HRQOL questionnaire validated to assess QOL in ESRD on HD patients. ${ }^{1,4}$ It is reliable and recommended by the National Kidney Foundation, ${ }^{3}$ and it consists of 36 items describing the perception of health state during the last 4 weeks leading to a five-dimension profile on a 100point scale, such as burden of kidney disease, symptoms and 
problems, effects of kidney disease on daily life, MCS, and PCS; a higher score indicates better perceived health state. ${ }^{2}$

\section{Results}

A total of 111 ESRD patients with a mean age of $61.8( \pm 15.5)$ years on HD were included, and there were more males than females (64.9\% vs $35.1 \%)$; the mean time-on-dialysis at the time of the study was 4.3 (4.8) years. Most were Caucasian (45\%), African-American (21.6\%), Hispanic (24.3\%), Asian, and others (10\%). Intradialytic weight gain average was $2.79 \mathrm{~kg}$ (standard deviation \pm 0.98 ) and hemoglobin $10.4 \mathrm{~g} / \mathrm{dL}$ (Tables 1 and 2). The KDQOL scores were reported on an average of $99 \%$ in all five domains (burden of kidney disease, symptoms and problems, effects of kidney disease on daily life, MCS, PCS). The scores for each domain were classified in categories of below average, average, and above average after being compared to the most recent national data using the KDQOL website. Approximately two-thirds of the responses of the questionnaire were ranked average. The remainders were split between above average and below average (Table 3).

Age, ethnicity, sex, intradialytic weight gain, URR, calcium, phosphorus, PTH, albumin, hemoglobin, transplant status, and vintage of dialysis were compared to the five components of KDQOL by chi-square and analysis of variance analyses. The $P$-value did not reach any statistical significance for all independent variables $(P>0.05)$. This was further confirmed by regression analysis (Tables 4 and 5). Of note, sex carried the strongest statistical significance (with a $P$-value of 0.16 ) as a predictor of "the burden of kidney disease on daily life" in ordinal regression.

\section{Discussion}

QOL has become a recommended clinical tool to assess patients on HD and a primary end point for multiple studies to describe the effectiveness of overall disease management. ${ }^{4}$

Table I Population demographics and characteristics

\begin{tabular}{ll}
\hline Variable & Mean ( \pm SD)/frequency (\%) \\
\hline $\begin{array}{l}\text { Age, years } \\
\text { Sex }\end{array}$ & $61.86( \pm 15.50)$ \\
$\quad$ Female & $39(35.1 \%)$ \\
$\quad$ Male & $72(64.9 \%)$ \\
Ethnicity & \\
$\quad$ African American & $24(21.6 \%)$ \\
$\quad$ Caucasian & $50(45.0 \%)$ \\
Hispanic & $27(24.3 \%)$ \\
Asian & $7(6.3 \%)$ \\
$\quad$ Others & $3(2.7 \%)$ \\
\hline
\end{tabular}

Abbreviation: SD, standard deviation.
Table 2 Descriptive statistics

\begin{tabular}{lllll}
\hline Variable & Minimum & Maximum & Mean & SD \\
\hline Wt gain, kg & 0.2 & 5.5 & 2.799 & $0.985 \mathrm{I}$ \\
URR, \% & 53 & 84 & 70.53 & 5.145 \\
Calcium, mg/dL & 6.2 & 10.9 & 8.984 & 0.6864 \\
$\mathrm{PO}_{4}, \mathrm{mg} / \mathrm{dL}$ & 2.8 & 11.0 & 5.289 & 1.6676 \\
$\mathrm{PTH}, \mathrm{pg} / \mathrm{mL}$ & 33 & 2.458 & 524.50 & 458.066 \\
Albumin, mg/dL & 2.2 & 4.5 & 3.886 & 0.3521 \\
Hemoglobin, g/dL & 6.7 & 14.2 & $10.40 \mathrm{I}$ & 1.3438 \\
Vintage of dialysis, years & 0.2 & 22.0 & 4.280 & 4.8477 \\
\hline
\end{tabular}

Abbreviations: SD, standard deviation; $\mathrm{Wt}$, weight; PTH, parathyroid hormone; URR, urea reduction ratio.

ESRD is associated with many comorbid conditions and require close medical management and follow-up. These facts can cause severe stress and significantly affect patient's overall physical and mental well-being. Patients have to deal with a strict nutritional regimen, debilitating physical complaints, social challenges, and psychological distress attributed to the often debilitating aspect of ESRD. ${ }^{8}$

A better HRQOL usually reflects to a better overall control of the disease (lesser symptoms and fewer handicaps due to the disease and its treatment). Multiple studies have hypothesized that comorbidities, physical symptoms, mental health, and physical conditioning form an intertwined web that ultimately affect QOL. ${ }^{6}$ Hemoglobin seems to be the only independent contributing factor. ${ }^{6}$

Different studies have been conducted looking for correlation between the metabolic profile and the QOL in patients on HD, and physical well-being seems to be positively affected by lower protein intake and higher creatinine values, ${ }^{3}$ while low serum albumin and creatinine values, which reflect protein energy wasting, are predictably associated with worse QOL. Also, people with obesity report significant lower QOL. In our study, neither the albumin nor the creatinine levels correlated with better physical activity score. Thus, interventions that improve protein energy intake without increasing body fat percentage need to be explored. ${ }^{4}$ On the other hand, hemoglobin level in our population was $10.4 \pm 1.3 \mathrm{~g} / \mathrm{dL}$ and achieved the recommended target and did not predict the physical functioning. As reported by Jaar et al, the physical functioning improved when anemia was partially corrected with the use of erythropoietin-stimulating agents (ESAs); however, maximizing therapy targeting higher hemoglobin levels was not accompanied by a parallel improvement of HRQOL and was associated with the increased risk of stroke. ${ }^{1}$

In addition, depression, a well-known condition to increase disease burden, was associated with morning shift dialysis, anemia, and phosphorus levels, which independently 
Table 3 Distribution of scores for the five components of KDQOL

\begin{tabular}{|c|c|c|c|c|c|}
\hline Frequency (\%) & Burden of kidney disease & Symptoms and problems & $\begin{array}{l}\text { Effects of kidney disease } \\
\text { on daily life }\end{array}$ & MCS & PCS \\
\hline $\mathrm{BA}$ & $14(12.8)$ & $15(23.5)$ & $19(17.1)$ & $16(14.5)$ & $10(9.1)$ \\
\hline A & $75(68.8)$ & $70(63.1)$ & $72(64.9)$ & $73(66.4)$ & $74(67.3)$ \\
\hline AA & $20(18.3)$ & $26(23.4)$ & $20(18)$ & $21(19.1)$ & $26(23.6)$ \\
\hline
\end{tabular}

Abbreviations: KDQOL, Kidney Disease QOL-36 surveys; BA, below average; A, average; AA, above average; MCS, mental component survey; PCS, physical component survey.

predicted the depression in ESRD population. ${ }^{9}$ Other factors that seem to affect mental well-being are lower calcium levels and absence of diabetes mellitus. ${ }^{3}$

Sleep quality in ESRD population is significantly related to phosphorus and albumin levels and body mass index. ${ }^{10}$ Moreover, keeping serum potassium between 3.5 and $5 \mathrm{mEq} / \mathrm{L}$ correlated with better QOL, ${ }^{11}$ while higher serum phosphorus level was associated with worse pruritus, ${ }^{12,13}$ and with increased hospitalization which in turned reflected worse QOL. ${ }^{14}$

Factors that seem to be associated with QOL in some studies have not been found to be consistent predictors in other studies. Mazairac et al did not find a significant association between hemogolobin, PTH, presence of arteriovenous fistula, $\mathrm{Kt} / \mathrm{V}$, phosphorus, blood pressure, and all 14 HRQOL domains. ${ }^{15}$

Intradialytic weight change seems to be a novel predictor of QOL. Taskapan et al found that higher IDWC (intradialytic weight change) negatively affected the vitality and social function domains of the QOL. They also found that people with depression had higher IDWC. ${ }^{16}$ Greater IDWC was also associated with longer recovery times form a dialysis session, which can be used to identify people with worse HRQOL. ${ }^{17}$ In addition, patients with lower satisfaction with perceived social support and with one or more comorbidities had higher IDWC.${ }^{18}$ However, in our study, the IDWC was not significantly different among all domains of KDQOL after scores classification (significance $>0.05$ ).
People respond to their disease by creating a unique perception of their condition in order to help them deal with its related challenges. ${ }^{8}$ Patients' beliefs, disease perception, and understanding of treatment modalities directly affect QOL and psychological well-being. ${ }^{6,8}$ Patients with the most social support and spiritual beliefs were less hospitalized and had better HRQOL when compared with those having poor social support. Interventions that aim to provide resources and social support and enhance independence need to be investigated. ${ }^{8}$

In our retrospective chart review study, we studied the predictive value of the metabolic profile and other independent variables for the QOL scores in its five domains. No relationships were statistically significant between the five dependent variables of interest (burden of kidney disease, symptoms and problems, effects on kidney disease on daily life, MCS, PCS) and the independent variables (age, ethnicity, sex, weight gain, calcium, URR, $\mathrm{PO}_{4}, \mathrm{PTH}$, albumin, hemoglobin, years on dialysis). These results put into discussion the validity of the metabolic profile as surrogate markers of the subject well-being. The biologic profile could not reflect the overall physical and mental burden, and we believe that the control of ESRD treatment challenges blunted any correlation and emphasize the importance of a multidisciplinary team approach in order to achieve a better QOL in the ESRD population by individually controlling all these factors. ${ }^{3}$

Table 4 Significance by ANOVA

\begin{tabular}{|c|c|c|c|c|c|}
\hline \multirow[t]{2}{*}{ Variable } & \multicolumn{5}{|l|}{ Significance } \\
\hline & Burden of kidney disease & Symptoms and problems & $\begin{array}{l}\text { Effects of kidney disease } \\
\text { on daily life }\end{array}$ & MCS & PCS \\
\hline Age & 0.388 & 0.340 & 0.439 & 0.147 & 0.331 \\
\hline Wt gain & 0.756 & 0.352 & 0.755 & 0.300 & 0.693 \\
\hline URR & 0.259 & 0.794 & 0.956 & 0.247 & 0.088 \\
\hline Calcium & 0.099 & 0.286 & 0.101 & 0.145 & 0.663 \\
\hline $\mathrm{PO}_{4}$ & 0.229 & 0.534 & 0.164 & 0.659 & 0.752 \\
\hline PTH & 0.054 & 0.599 & 0.584 & 0.178 & 0.642 \\
\hline Albumin & 0.907 & 0.082 & 0.949 & 0.781 & 0.363 \\
\hline Hemoglobin & 0.140 & 0.698 & 0.252 & 0.919 & 0.578 \\
\hline Yrs on dialysis & 0.958 & 0.921 & 0.246 & 0.327 & 0.185 \\
\hline
\end{tabular}

Note: The bold value was close to statistical significance.

Abbreviations: ANOVA, analysis of variance; MCS, mental component survey; PCS, physical component survey; Wt, weight; PTH, parathyroid hormone; Yrs, years; URR, urea reduction ratio. 
Table 5 Pearson chi-square analysis

\begin{tabular}{llllr}
\hline Variable & \multicolumn{2}{l}{ Significance } & & \\
\cline { 2 - 5 } & Burden of kidney disease & Symptoms and problems & \multicolumn{2}{l}{$\begin{array}{l}\text { Effects of kidney disease on MCS } \\
\text { daily life }\end{array}$} \\
\hline Sex & 0.500 & 0.826 & 0.642 & 0.254 \\
Transplant list status-attitude & 0.219 & 0.867 & 0.683 & 0.539 \\
Race & 0.768 & 0.580 & 0.922 & 0.127 \\
\hline
\end{tabular}

Abbreviations: MCS, mental component survey; PCS, physical component survey.

Although our population had comparable distribution to the national scores (Table 3 ), the study sample is small and may limit its ability to identify significant correlations. Also, we believe that the use of a precise and reliable method to measure the dietary protein intake and the report of marital and employment status in this analysis could have contributed to a more comprehensive results.

\section{Conclusion}

Improving patients' QOL has evolved as one of the primary goals of renal replacement therapy. ${ }^{4}$ Beyond dialysis prescriptions, there are several domains of poor performance that offer the opportunities to improve HRQOL in ESRD. Improving physical functioning and promoting independency could be an important perspective to change the disease perception and lessen its incapacitations.

More importantly, transplantation still remains the most effective form of renal replacement therapy for improving HRQOL, and it is an imperative modality to consider in managing patients. ${ }^{1}$

\section{Disclosure}

The authors report no conflicts of interest in this work.

\section{References}

1. Jaar BG, Chang A, Plantinga L. Can we improve quality of life of patients on dialysis? Clin J Am Soc Nephrol. 2013;8(1):1-4.

2. Pagels AA, Söderkvist BK, Medin C, Hylander B, Heiwe S. Health-related quality of life in different stages of chronic kidney disease and at initiation of dialysis treatment. Health Qual Life Outcomes. 2012;10:71.

3. Md Yusop NB, Yoke Mun C, Shariff ZM, Beng Huat C. Factors associated with quality of life among hemodialysis patients in Malaysia. PLoS One. 2013;8(12):e84152.

4. Feroze U, Noori N, Kovesdy CP, et al. Quality-of-life and mortality in hemodialysis patients: roles of race and nutritional status. Clin JAm Soc Nephrol. 2011;6(5):1100-1111.
5. Wu AW, Fink NE, Marsh-Manzi JV, et al. Changes in quality of life during hemodialysis and peritoneal dialysis treatment: generic and disease specific measures. J Am Soc Nephrol. 2004;15(3):743-753.

6. Chan R, Brooks R, Erlich J, et al. How do clinical and psychological variables relate to quality of life in end-stage renal disease? Validating a proximal-distal model. Qual Life Res. 2014;23(2):677-686.

7. Lægreid IK, Aasarød K, Bye A, Leivestad T, Jordhøy M. The impact of nutritional status, physical function, comorbidity and early versus late start in dialysis on quality of life in older dialysis patients. Ren Fail. 2014;36(1):9-16.

8. Theofilou P. Quality of life and mental health in hemodialysis and peritoneal dialysis patients: the role of health beliefs. Int Urol Nephrol. 2012;44(1):245-253.

9. Teles F, Azevedo VF, Miranda CT, Miranda MP, Teixeira Mdo C, Elias RM. Depression in hemodialysis patients: the role of dialysis shift. Clinics (Sao Paulo). 2014;69(3):198-202.

10. Emami Zeydi A, Jannati Y, Darvishi Khezri H, et al. Sleep quality and its correlation with serum C-reactive protein level in hemodialysis patients. Saudi J Kidney Dis Transpl. 2014;25(4):750-755.

11. Yamana E. The relationship of clinical laboratory parameters and patient attributes to the quality of life of patients on hemodialysis. Jpn J Nurs Sci. 2009;6(1):9-20.

12. Chiu YW, Teitelbaum I, Misra M, de Leon EM, Adzize T, Mehrotra R. Pill burden, adherence, hyperphosphatemia, and quality of life in maintenance dialysis patients. Clin J Am Soc Nephrol. 2009;4(6): 1089-1096.

13. Gatmiri SM, Mahdavi-Mazdeh M, Lessan-Pezeshki M, Abbasi M. Uremic pruritus and serum phosphorus level. Acta Med Iran. 2013; 51(7):477-481.

14. Rostami Z, Hosseini MS, Lessan Pezeshki M, Heidari F, Einollahi B. Bone mineral metabolism and subsequent hospitalization with poor quality of life in dialysis patients. Nephrourol Mon. 2014;6(1):e14944.

15. Mazairac AH, de Wit GA, Grooteman MP, et al. Clinical performance targets and quality of life in hemodialysis patients. Blood Purif. 2012;33(1-3):73-79.

16. Taskapan H, Ates F, Kaya B, et al. Psychiatric disorders and large interdialytic weight gain in patients on chronic haemodialysis. Nephrology (Carlton). 2005;10(1):15-20.

17. Rayner HC, Zepel L, Fuller DS, et al. Recovery time, quality of life, and mortality in hemodialysis patients: the Dialysis Outcomes and Practice Patterns Study (DOPPS). Am J Kidney Dis. 2014;64(1):86-94.

18. Pang SK, Ip WY, Chang AM. Psychosocial correlates of fluid compliance among Chinese haemodialysis patients. J Adv Nurs. 2001;35(5): 691-698.

\section{Publish your work in this journal}

The International Journal of Nephrology and Renovascular Disease is an international, peer-reviewed open-access journal focusing on the pathophysiology of the kidney and vascular supply. Epidemiology, screening, diagnosis, and treatment interventions are covered as well as basic science, biochemical and immunological studies. The journal welcomes

\section{Dovepress}

original research, clinical studies, reviews \& evaluations, expert opinion and commentary, case reports and extended reports. The manuscript management system is completely online and includes a very quick and fair peerreview system, which is all easy to use. Visit http://www.dovepress.com/ testimonials.php to read real quotes from published authors. 